\title{
Variants of the Aortic Arch in Adult General Population and their Association with Thoracic Aortic Aneurysm Disease
}

\author{
Sameh Yousef ${ }^{1}$, saket singh ${ }^{2}$, Abedalrazaq AlKukhun ${ }^{2}$, Bilal Alturkmani ${ }^{2}$, Makoto Mori ${ }^{2}$, \\ Julia Chen ${ }^{3}$, Clancy Mullan ${ }^{1}$, Cornell Brooks ${ }^{1}$, Roland Assi ${ }^{2}$, Peter Gruber ${ }^{2}$, Isabel \\ Cortopassi $^{2}$, Arnar Geirsson $^{2}$, and Prashanth Vallabhajosyula ${ }^{1}$ \\ ${ }^{1}$ Yale School of Medicine \\ ${ }^{2}$ Yale University School of Medicine \\ ${ }^{3}$ Yale-New Haven Hospital
}

January 4, 2021

\begin{abstract}
Background. Query a single institution computed tomography (CT) database to assess the prevalence of aortic arch anomalies in general adult population and their potential association with thoracic aortopathies. Methods. CT chest scan reports of patients aged 50-85 years old performed for any indication at a single health system between 2013 and 2016 were included in the analysis. Characteristics of patients with and without aortic arch anomalies were compared by t-test and Fisher exact tests. Logistic regression analysis was performed to assess for independent risk factors of thoracic aortic aneurysm. Results. Of 21,336 CT scans, 603 (2.8\%) described arch anomalies. Bovine arch $(\mathrm{n}=354,58.7 \%)$ was the most common diagnosis. Patients with arch anomalies were more likely to be female $(\mathrm{p}<0.001)$, non-Caucasian $(\mathrm{p}<0.001)$, and hypertensive $(\mathrm{p}<0.001)$. Prevalence of thoracic aortic aneurysm in arch anomalies group was $10.8 \%(\mathrm{n}=65)$ compared to $4.1 \%(\mathrm{n}=844)$ in the non-arch anomaly cohort $(\mathrm{p}<0.001)$. The highest prevalence of thoracic aneurysm was associated with right-sided arch combined with aberrant left subclavian configuration (33\%), followed by bovine arch (13\%), and aberrant right subclavian artery (8.2\%). On binary logistic regression, arch anomaly $(\mathrm{OR}=2.85$ [2.16-3.75]), aortic valve pathology (OR 2.93 [2.31-3.73]), male sex (OR 2.38 [2.01$2.80]$ ), and hypertension (OR 1.47 [1.25-1.73]) were significantly associated with increased risk of thoracic aneurysm disease. Conclusions. Reported prevalence of aortic arch anomalies by CT imaging in the older adult population is $\sim 3 \%$, with high association of thoracic aortic aneurysm $(\mathrm{OR}=2.85)$ incidence in this subgroup. This may warrant a more tailored surveillance strategy for aneurysm disease in this subpopulation.
\end{abstract}

\section{Introduction}

Aortic arch anomalies constitute a broad spectrum of congenital variants of the development of the aortic arch and great vessels. During embryology, bilateral ventral and dorsal aortae are connected by arches, most of them regress except for the $3^{\text {rd }}$ arch that gives rise to the carotid arteries, the $4^{\text {th }}$ arch that gives rise to the adult arch, and the $6^{\text {th }}$ arch that gives rise to the ductus arteriosus ${ }^{1}$. Due to this complex developmental process, variations or anomalies of the arch and its branches may occur ${ }^{2}$. Anomalies of the origin of arch vessels include common origin of the left common carotid and the brachiocephalic arteries (bovine arch) ${ }^{3}$, aberrant origin of the right and left subclavian arteries from the descending aorta that sometimes originate from a localized dilatation called Kommerell's diverticulum ${ }^{4,5}$, and aberrant origin of the left vertebral artery from the arch itself rather than originating from the left subclavian artery ${ }^{6}$. Anomalies of the laterality and position of the arch includes double arch, right sided arch, circumflex arch, cervical arch, interrupted arch, and pseudocoarctation 7,8 . Some variants are reported to be very common in the general population, with example bovine arch reported to have a prevalence of $\sim 20 \%$, while other variants such as double and cervical arch patterns are rare ${ }^{2}$. It has been reported that arch anomalies are associated with increased incidence 
of congenital heart disease ${ }^{9}$ and also thoracic aortopathies ${ }^{10}$, although large population studies on arch anomalies are lacking. We assessed our institutional CT scan database to better understand the prevalence of arch anomalies in the older adult population age $>50$ years, and its possible association with the thoracic aortic aneurysm disease.

\section{Methods}

Database and patient population . Institutional electronic medical record system was queried from years 2013-2016 to identify patients with age [?] 50 and $<85$ years who received CT chest imaging for either in inpatient, outpatient, or emergency department encounters. We set the lower age limit of 50 years because thoracic aortic aneurysm below this age is rare unless associated with connective tissue disease, and the upper age limit was set as the benefit of detection leading to intervention decreases at old age. After excluding multiple scans obtained on the same patient during the study period, there were 21,336 scans obtained on unique patients. In patients with multiple scans, the scan with the earliest date was used for the analysis. The Institutional Review Board approved this study. All CT scan reports were screened for any comments on the aortic arch and great vessels. Those reports were further reviewed for the description and subcategorization of arch anomalies. Also, all CT scans were screened for words and permutations related to thoracic aortic aneurysm (TAA) (aneurysm, dilation, dilatation, ectasia, enlargement) and reports containing such words were further reviewed for the site and the size of the aneurysms. Aneurysms of the abdominal aorta were excluded. The site of the thoracic aortic aneurysm was the site of the maximum diameter of the aorta. Subjects with missing clinical or demographic data were excluded.

Validation sample. Due to the concern that the aorta was not accurately assessed on scans done without contrast and routine scans done for non-thoracic pathology, a random sample of 200 scans was generated and reviewed by two radiology trainee and a board certified cardiothoracic radiologist with 10 years of experience in reading aorta studies. All the 200 scans were non-contrast. The prevalence and the type of the anomaly was documented, if found.

Patient characteristics . Indicated age, height, and weight values were recorded at the time of the CT scan. Race was categorized into Caucasian, African American, Asian, and other. Body surface area was calculated using Mosteller equation. ${ }^{11}$ Smoking history was dichotomized based on those with [?] 5 packyear smoking history indicating positive smoking history. Comorbidities (aortic valve disease, hypertension, diabetes, dyslipidemia, congestive heart failure, cocaine use, chronic kidney disease, myocardial infarction, chronic obstructive pulmonary disease) were chosen as commonly evaluated cardiovascular comorbidities and were defined using ICD-10 codes. To define aortic dilatation and aneurysm; $4 \mathrm{~cm}$ cut-off value was used for the root, ascending and the aortic arch, and $3 \mathrm{~cm}$ was used for the descending thoracic aorta.

Statistical analysis. Differences in the patient characteristics were compared with two-tailed t-test for normally distributed continuous variables and Fisher's exact test for categorical variables. Multivariable logistic regression model was fitted to identify independent risk factors of TAA. Age, sex, race, BSA, history of hypertension, diabetes, dyslipidemia, smoking history, COPD, arch anomalies and aortic valve diseases were included in the model. $\mathrm{P}$ value of $<0.05$ and $95 \%$ CI were used to define statistically significant difference. Analysis was conducted using Microsoft excel 2019 and Prism 8.2 (GraphPad Software, San Diego, CA) for simple analysis and SAS 9.4 (SAS Institute Inc, Cary, NC) for modelling.

\section{Results}

In this cohort of 21,336 unique patients aged 50-85 years who underwent CT including the chest (49.3\% $(\mathrm{n}=10,508)$ were CT scans with intravenous contrast and $2.5 \%(\mathrm{n}=540)$ were EKG-gated $)$ for any clinical indication, arch anomalies were reported in $2.8 \%(\mathrm{n}=603$ patients). Bovine arch was the most common variant ( $\mathrm{n}=354,58.7 \%$ of all anomalies), followed by aberrant right subclavian artery ( $\mathrm{n}=147,24.4 \%)$, aberrant left vertebral artery $(\mathrm{n}=95,15.8 \%)$, aberrant left subclavian artery combined with right sided arch $(\mathrm{n}=12,2 \%)$, double arch $(\mathrm{n}=1,0.2 \%)$, pseudocoarctation $(\mathrm{n}=1,0.2 \%)$ (Figure 1). Aberrant left subclavian artery arising from Kommerell's diverticulum was noted in 5 patients and aberrant right subclavian artery arising from Kommerell's diverticulum was reported in 3 patients. On bivariate analysis, patients with 
arch anomalies were more likely to be females $(\mathrm{p}<0.001)$, non-Caucasian $(\mathrm{p}<0.001)$, hypertensive $(\mathrm{p}<0.001)$, diabetic $(\mathrm{p}=0.012)$, had hyperlipidemia $(\mathrm{p}=0.037)$, and with significantly higher prevalence of aortic valve disease $(\mathrm{p}<0.001)$ and TAA $(\mathrm{p}<0.001)$. Patients with arch anomalies less commonly had history of smoking and COPD (Table1).

The prevalence of TAA disease was different according to the type of arch anomaly (Figure 3). Subjects with aberrant left subclavian artery combined with right sided arch had the highest prevalence of TAA (33\% versus $4.4 \% ; 4$ out of 12 patients; $\mathrm{P}<0.001)$, followed by bovine arch $(13 \%$ versus $4.4 \%$;; 46 out of 354 patients with bovine aortic arch; $\mathrm{P}<0.001)$, and aberrant right subclavian artery ( $8 \%$ versus 4.4\%; 12 out of 147 patients with aberrant right subclavian artery; $\mathrm{P}<0.001$ ). Aberrant left vertebral artery was not associated with increased prevalence of TAA (3\% versus $4.4 \% ; 3$ out of 95 patients with aberrant left vertebral artery; $\mathrm{P}=0.99$ ). The regional distribution of TAA was not different between the arch anomaly group and the no anomaly group. TAA in both groups were more likely to affect the ascending aorta $>$ descending aorta $>$ aortic root $>$ aortic arch (Figure 2). The aortic diameter in the respective region was also not different between the 2 groups. In the region of the root: mean aortic diameter was $4.2 \pm 0.3 \mathrm{~cm}$ versus $4.3 \pm 0.4 \mathrm{~cm}, \mathrm{P}=0.68$; in the ascending aorta: $4.3 \pm 0.3 \mathrm{~cm}$ versus $4.3 \pm 0.4 \mathrm{~cm}, \mathrm{P}=0.92$; in the arch: $4.6 \pm 0.5 \mathrm{~cm}$ versus $4.6 \pm 0.7 \mathrm{~cm}, \mathrm{P}=0.96$; and in the descending aorta: $3.6 \pm 0.6 \mathrm{~cm}$ versus $3.7 \pm 0.8 \mathrm{~cm}$, $\mathrm{P}=0.60$. In the validation sample, 49 cases were previously known to have TAA and were excluded from the analysis. In the remaining 151 scans, 32 patients were found to have bovine arch (prevalence=21\%). Type 1 arch was present in 28 cases and type 2 was present in 4 cases. Also, the aberrant left vertebral artery was encountered in 10 patients (6.6\%). Other anomalies were not encountered in the validation sample.

To define independent risk factors for TAA and dilatations, a multivariable logistic regression analysis was performed. The model included patient's age, male sex, Caucasian race, BSA, smoking, hypertension, hyperlipidemia, COPD, aortic valve disease, and arch anomalies. Age (OR = 1.04 CI[1.03-1.05]), male sex (OR $=2.38[2.01-2.80])$, BSA $(\mathrm{OR}=1.45[1.13-1.86])$, hypertension $(\mathrm{OR}=1.47[1.25-1.73])$, aortic valve disease $(\mathrm{OR}=2.93[2.31-3.73])$, and arch anomalies $(\mathrm{OR}=2.85[2.16-3.75])$ were independent risk factors for TAA (Figure 4).

\section{Comment}

In this study, we examined our clinical CT scan database to define arch anomalies, its variants, and the possible association with TAA. We found the reported prevalence of arch anomalies on initial survey to be $2.8 \%$, with bovine aortic arch being the most common anomaly $(58.7 \%$ of all anomalies and $1.6 \%$ in this population), and aberrant left subclavian artery combined with right sided arch to have the highest association with TAA $(33 \%, \mathrm{P}<0.001){ }^{12-14}$. The reported prevalence of $2.8 \%$ of arch anomalies was much lower than what we found in the validation sample $(21 \%$ for bovine arch and $6.6 \%$ for aberrant left vertebral artery). The results of the validation sample correspond to previously published reports on the prevalence of bovine arch anomaly. ${ }^{2,16}$ This deviation from the literature in the initial survey is due to under reporting in our clinical database, as the CT scans included in this study were done for indications other than aorta diseases (such as lung cancer screening, pulmonary diseases, infection/ sepsis, trauma, pulmonary embolism, esophageal diseases) and it is possible that the arch anatomy received less attention from the reader since most of these cases were report by radiologists without fellowship training in cardiovascular imaging. With this relatively high prevalence of bovine arch, the clinical significance of it should be considered. Morrehead et al reported $31.1 \%$ prevalence of bovine arch, including $14.9 \%$ type I bovine arch, and $16.2 \%$ type II bovine arch in patients with thoracic aortic pathology. ${ }^{15}$ Dumfarth et al reported bovine aortic arch to be the most common anomalous branch pattern in patients with thoracic aortic disease, with a prevalence of $24.6 \%$. This was followed by isolated left vertebral artery in $6.3 \%$ of patients with thoracic aortic disease, and an aberrant right subclavian artery in $1.8 \%{ }^{18}$ Wanamaker et al., reported similar finding with bovine arch being the most common association with aortic dissection followed by isolated left vertebral artery. ${ }^{10}$

We also noticed that patients with arch anomalies had higher association with aortic valve disease and hypertension. It is difficult to know if one factor predisposes the occurrence of the other but we can hypothesize that it is also possible that fewer /anomalous branching of the aorta in this region leads to abnormal flow 
dynamics throughout the aortic wall in this region putting it under abnormal hemodynamic stress which when combined with other factors like hypertension or wall stress caused by abnormal aortic valve geometry could result in an aggressive phenotype leading to aortic dilatation and even dissection due to the added/synergistic effect of multiple wall stressors. ${ }^{19}$

Given the nearly 3 fold higher association with TAA in the arch anomaly subgroup compared to subjects without arch anomalies in this cohort and also from the forementioned reports, greater attention needs to be paid to carefully look and document for bovine arch anatomy in patients undergoing routine CT chest imaging, with follow-up over time for development of TAA. To some extent, the argument for this is similar to incidental diagnosis of bicuspid aortic valve congenital variant, which occurs in $\sim 1-2 \%$ of the general population. These patients are carefully monitored over time for development of aortic valvulopathy and ascending aortopathy, although majority of subjects with bicuspid aortic valve will not need any surgical intervention.

The prevalence of other aortic arch variants noted in our study is in accordance to that reported in the literature, which is $\sim 1-2 \%{ }^{17}$. Our study also shows differential influence of the type of arch anomaly on the size of the aorta. Right sided aortic arch combined with aberrant left subclavian had the highest association with TAA (33\%), while aberrant left vertebral artery was not associated with increased risk of TAA (3\%).

In this cohort, we also defined independent risk factors for TAA and found significant association of arch anomaly with incidental aortic aneurysm (OR 2.85). Other independent factors were: aortic valve disease $(\mathrm{OR}=2.95)$, age, male sex, and increased BSA, while diabetes was found to be protective $(\mathrm{OR}=0.75)$. Given the strong association of arch anomalies with TAA in this study and also in the literature, and the fact that TAA in this sub-population tends to dissect more often ${ }^{4,5}$, we suggest further investigation for inclusion of aortic arch anomalies in the guidelines for screening for TAA. Current guidelines for screening and follow-up of patients with known thoracic aortic disease does not take into consideration the presence of aortic arch anomalies ${ }^{20,21}$.

\section{Limitations}

The study defined aortic arch anomalies in patients undergoing CT scan chest for variety of indications at a single institution. Most of these indications were for non-cardiac/vascular reasons. While this eliminates investigator bias, we noticed that CT scans read by radiologists who are not fellowship trained in cardiovascular imaging underreport the presence of arch anomalies, particularly the "bovine arch" variant. The study included all types of CT scans (contrast CT scans $=49.3 \%(\mathrm{n}=10,508)$ and EKG gated scans $=2.5 \%(\mathrm{n}=540)$ ) which allowed for a larger sample size, but might have resulted into significantly lower rates of the reported anomalies, which are known to be better visualized using contrast studies. We used a size threshold for diagnosing TAA (4 cm as cut-off value for defining the root, ascending and arch dilatations/aneurysms and $3 \mathrm{~cm}$ for the descending aorta) as aortic size continues to be the initial screening tool for TAA by most radiologists and physicians who can further refer the patients for appropriate consultation. Although, the impact of normalization to body characteristics (age, sex, BSA) in this study is unknown and might influence the prevalence of TAA. The study did not address the prevalence of arch anomalies in patients with thoracic aortic dissection as the number of dissection patients in this database was too low to analyze $(\mathrm{n}=32)$.

\section{Conclusions}

This study investigated the reported prevalence of arch anomalies and its variants in the adult general population (50 to 85 years of age) undergoing CT chest imaging at a tertiary care center. Arch anomalies were under-reported and the high association with TAA in this subpopulation (OR 2.85) may warrant more methodological approach in reviewing the arch pattern and also more periodic imaging surveillance and patient counselling in patients with aortic arch anomalies.

\section{References}

1. Sissman NJ. Developmental landmarks in cardiac morphogenesis: comparative chronology. The American journal of cardiology.1970;25(2):141-8. 
2. Kau T, Sinzig M, Gasser J, et al. Aortic development and anomalies. Semin Intervent Radiol. 2007 Jun;24(2):141-52.

3. Layton KF, Kallmes DF, Cloft HJ, Lindell EP, Cox VS. Bovine aortic arch variant in humans: clarification of a common misnomer. AJNR Am J Neuroradiol. 2006 Aug;27(7):1541-2.

4. Kommerell B. Verlagerung des osophagus durch eine abnorm verlaufende arteria subclavia dextra (arteria lusoria). Fortschr Roentgenster. 1936;54:590-5.

5. van Son JA, Konstantinov IE. Burckhard F. Kommerell and Kommerell's diverticulum. Tex Heart Inst J. 2002;29(2):109-12.

6. Suzuki S, Kuwabara Y, Hatano R, Iwai T. Duplicate origin of left vertebral artery.Neuroradiology. 1978 Mar 29;15(1):27-9.

7. McElhinney DB, Thompson LD, Weinberg PM, Jue KL, Hanley F. Surgical approach to complicated cervical aortic arch: anatomic, developmental, and surgical considerations.Cardiol Young. 2000 May;10(3):212-9.

8. Cinà CS, Althani H, Pasenau J, Abouzahr L. Kommerell's diverticulum and right-sided aortic arch: a cohort study and review of the literature. J Vasc Surg. 2004 Jan;39(1):131-9.

9. Hastreiter AR, D'Cruz IA, Cantez T, Namin EP, Licata . Right-sided aorta. I. Occurrence of right aortic arch in various types of congenital heart disease. II. Right aortic arch, right descending aorta, and associated anomalies. British heart journal. 1966;28(6):722.

10. Wanamaker KM, Amadi CC, Mueller JS, Moraca R. Incidence of aortic arch anomalies in patients with thoracic aortic dissections. J Card Surg. 2013 Mar;28(2):151-4.

11. Mosteller RD. Simplified calculation of body-surface area. N Engl J Med. 1987 Oct 22;317(17):1098.

12. Naoum JJ, Parenti JL, LeMaire SA, Coselli JS. Endovascular repair of a right-sided descending thoracic aortic aneurysm with a right-sided aortic arch and aberrant left subclavian artery. Ann Thorac Surg. 2008 Mar;85(3):1074-6.

13. Reid DG. Three Examples of a Right Aortic Arch. J Anat Physiol. 1914 Jan;48(Pt 2):174-81.

14. Caus T, Gaubert JY, Monties JR, et al. Right-sided aortic arch: surgical treatment of an aneurysm arising from a Kommerell's diverticulum and extending to the descending thoracic aorta with an aberrant left subclavian artery.Cardiovasc Surg. 1994 Feb;2(1):110-3.

15. Moorehead PA, Kim AH, Miller CP, Kashyap TV, Kendrick DE, Kashyap VS. Prevalence of Bovine Aortic Arch Configuration in Adult Patients with and without Thoracic Aortic Pathology. Ann Vasc Surg. 2016 Jan;30:132-7.

16. Hornick M, Moomiaie R, Mojibian H, et al. 'Bovine' aortic arch - a marker for thoracic aortic disease.Cardiology. 2012;123(2):116-24.

17. Türkvatan A, Büyükbayraktar FG, Olçer T, Cumhur T. Congenital anomalies of the aortic arch: evaluation with the use of multidetector computed tomography. Korean J Radiol.2009 Mar-Apr;10(2):176-84.

18. Dumfarth J, Chou AS, Ziganshin BA, et al. Atypical aortic arch branching variants: a novel marker for thoracic aortic disease. The Journal of thoracic and cardiovascular surgery. 2015;149(6):1586-92.

19. Wagenseil JE. Bio-chemo-mechanics of thoracic aortic aneurysms. Curr Opin Biomed Eng. 2018;5:50-7.

20. Erbel R, Aboyans V, Boileau C, et al. 2014 ESC Guidelines on the diagnosis and treatment of aortic diseases: Document covering acute and chronic aortic diseases of the thoracic and abdominal aorta of the adult. The Task Force for the Diagnosis and Treatment of Aortic Diseases of the European Society of Cardiology (ESC). Eur Heart J. 2014 Nov 1;35(41):2873-926.

21. Hiratzka LF, Bakris GL, Beckman JA, et al 2010 ACCF/AHA/AATS/ACR/ASA/SCA/SCAI/SIR/STS/SVM Guidelines for the diagnosis and management of patients with thoracic aortic disease. A Report of the American College of Cardiology Foundation/American Heart Association Task Force on Practice Guidelines, American Association for Thoracic Surgery, American College of Radiology,American Stroke Association, Society of Cardiovascular Anesthesiologists, Society for Cardiovascular Angiography and Interventions, Society of Interventional Radiology, Society of Thoracic Surgeons,and Society for Vascular Medicine. J Am Coll Cardiol. 2010 Apr 6;55(14):e27-e129.

\section{Legend to Figures}


Figure 1. Distribution of aortic arch anomaly variants is shown.

Figure 2. Anatomic distribution of TAA in subjects with and without arch anomalies is shown.

Figure 3. Percentage of subjects with concomitant TAA for each arch anomaly variant is shown

Figure 4. Multivariable regression analysis of 21,336 subjects was performed to assess for risk factors associated with incidental TAA. Arch anomaly was significantly associated with TAA incidence (OR 2.85).

OR: odds ratio, CI: 95\% confidence interval, AV: aortic valve, COPD: chronic obstructive pulmonary disease, BSA: body surface area.

Table 1 . Characteristics of patients with and without arch anomalies.

\begin{tabular}{|c|c|c|c|c|}
\hline & & $\begin{array}{l}\text { Anomalies } \mathrm{n}=603 \\
(2.8 \%)\end{array}$ & $\begin{array}{l}\text { No anomalies } \\
\mathrm{n}=20733(97.2 \%)\end{array}$ & $\mathrm{P}$ \\
\hline $\begin{array}{l}\text { Age (years) } \pm \\
\text { SD }\end{array}$ & $\begin{array}{l}\text { Age (years) } \pm \\
\text { SD }\end{array}$ & $66 \pm 9.2$ & $66.6 \pm 9.3$ & 0.154 \\
\hline Male sex & Male sex & $253(42)$ & $11722(56.5)$ & $<0.001$ \\
\hline BSA $\left(m^{2}\right)$ & BSA $\left(m^{2}\right)$ & $1.9 \pm 0.3$ & $1.89 \pm 0.3$ & 0.104 \\
\hline \multirow[t]{5}{*}{ Race } & Caucasian & $449(74.5)$ & $16643(80.3 \%)$ & $<0.001$ \\
\hline & African & $84(13.9)$ & $2186(10.5)$ & \\
\hline & American & & & \\
\hline & Asian & $3(0.5)$ & $311(1.5)$ & \\
\hline & Other & $67(11.1)$ & $1587(7.7)$ & \\
\hline Smoking & Smoking & $233(38.6)$ & $8969(43.3)$ & 0.024 \\
\hline Hypertension & Hypertension & $405(67.2)$ & $12090(58.3)$ & $<0.001$ \\
\hline Hyperlipidemia & Hyperlipidemia & $285(47.3)$ & $8911(43)$ & 0.037 \\
\hline Diabetes & Diabetes & $162(26.9)$ & $4651(22.4)$ & 0.012 \\
\hline Mellitus & Mellitus & & & \\
\hline CHF & CHF & $47(7.8)$ & $1757(8.5)$ & 0.603 \\
\hline Cocaine use & Cocaine use & 0 & $66(0.3)$ & 0.267 \\
\hline CKD & CKD & $35(5.8)$ & $1503(7.2)$ & 0.201 \\
\hline Previous MI & Previous MI & $41(6.8)$ & $1265(6.1)$ & 0.490 \\
\hline AV disease & AV disease & $41(6.8)$ & $249(1.2)$ & $<0.001$ \\
\hline MV disease & MV disease & $25(4.1)$ & $705(3.4)$ & 0.306 \\
\hline COPD & COPD & $80(13.3)$ & $3531(17)$ & 0.015 \\
\hline $\begin{array}{l}\text { Congenital } \\
\text { anomalies }\end{array}$ & $\begin{array}{l}\text { Congenital } \\
\text { anomalies }\end{array}$ & $13(2.2)$ & $265(1.3)$ & 0.067 \\
\hline TAA & TAA & $65(10.8)$ & $844(4.1)$ & $<0.001$ \\
\hline TAD & TAD & 0 & $32(0.2)$ & $>0.999$ \\
\hline
\end{tabular}

$\mathrm{BSA}=$ Body Surface Area, $\mathrm{CHF}=$ Congestive Heart Failure, CKD=Chronic Kidney Disease, $\mathrm{MI}=\mathrm{Myocardial}$ Infarction, $\mathrm{AV}=$ Aortic valve, $\mathrm{MV}=$ Mitral Valve, $\mathrm{COPD}=$ Chronic Obstructive Pulmonary Disease, $\mathrm{TAA}=$ Thoracic Aortic Aneurysm and TAD=Thoracic Aortic Dissection

\section{Hosted file}

Table 1.pdf available at https://authorea.com/users/352591/articles/502430-variants-ofthe-aortic-arch-in-adult-general-population-and-their-association-with-thoracic-aorticaneurysm-disease 


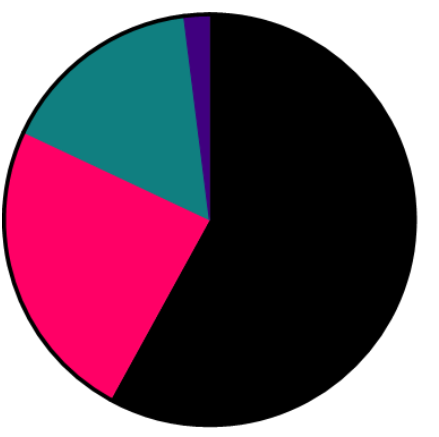

Bovine arch (58\%)

$\square$ Aberrant right subclavian artery $(24 \%)$

$\square$ Aberrant left vertebral artery (16\%)

- Aberrant left subclavian artery $(2 \%)$

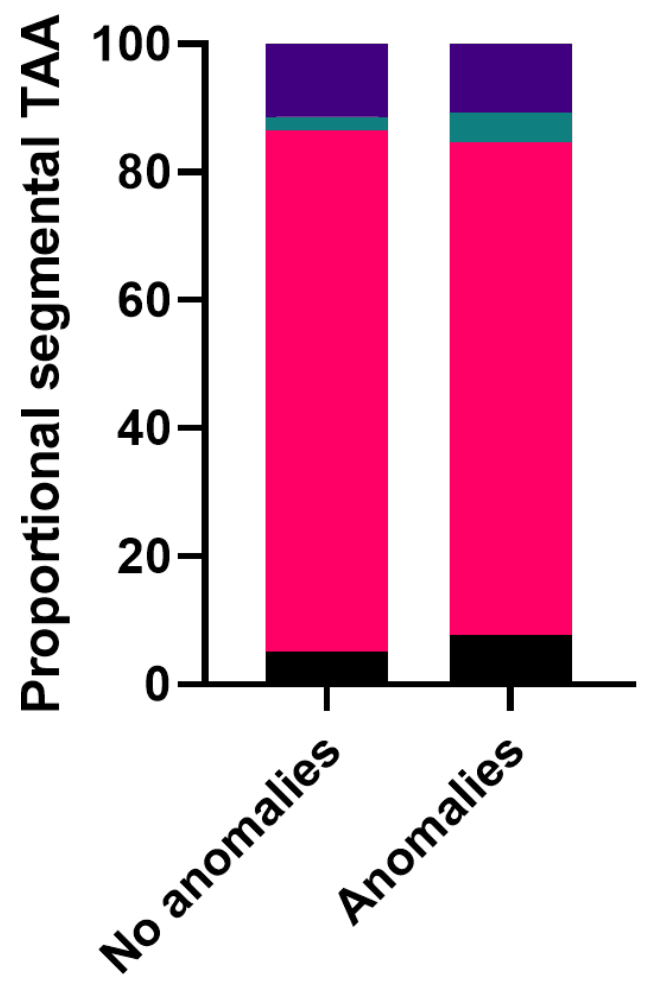

Descending

- Arch

- Ascending

noot

\section{Aortic arch configuration}

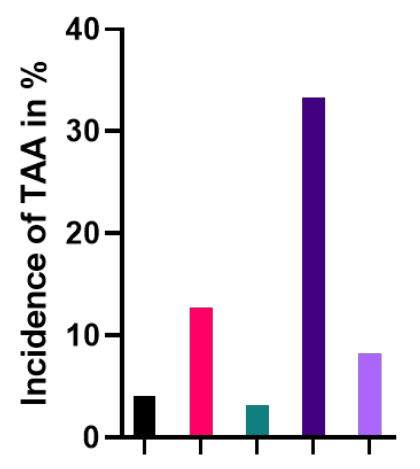

- No anomalies

bovine arch

- Aberrant left vertebral artery

- Aberrant left subclavian artery

- Aberrant right subclavian artery

Aortic arch configuration 


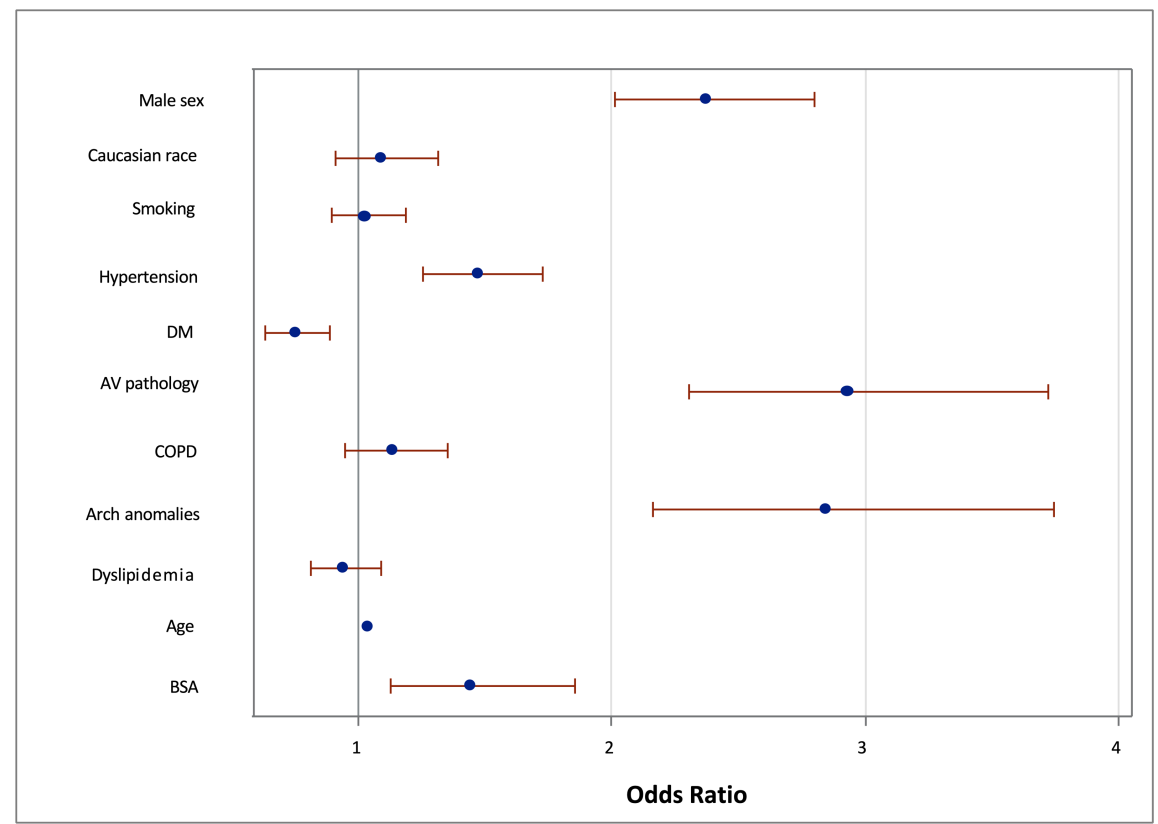

